\title{
Allergy to Montelukast Sodium Treated Effectively by Protracted Oral Desensitization: First Case Report
}

\section{Moufag Mohammed Saeed Tayeb}

King Abdul-Aziz University, Jeddah, Saudi Arabia

\begin{abstract}
Background: Antileukotrines such as Montelukast Sodium play a significant role in asthma control. IgE mediated hypersensitivity reactions to Montelukast are very rarely reported.

Objective: To explore the efficacy of oral desensitization in Montelukast hypersensitivity.

Methods: A 30 year old Saudi woman with uncontrolled asthma despite maximum pharmacological therapy presented to allergy clinic with recurrent symptoms of lip swelling, maculopapular skin rash and shortness of breath a few minutes after taking Montelukast $10 \mathrm{mg}$ orally. Eventually, she was diagnosed as a case of hypersensitivity reaction type I to Montelukast. As a consequence of her uncontrolled asthma symptoms and as no other alternative Antileukotrine was available, it was decided to start an oral desensitization with Montelukast. Phase I desensitization was conducted in the clinic by gradually increasing doses of oral Montelukast starting from $0.001 \mathrm{mg}$ up to $1 \mathrm{mg}$. Phase II desensitization was conducted at home with increasing doses starting from $1 \mathrm{mg}$ up to $10 \mathrm{mg} / \mathrm{day}$. Patient was followed by frequent clinic visits, emails and phone calls.

Results: Desensitization phases I was successful over a period of 3 hours with minimal reactions. Desensitization phase II was prolonged due to the occurrence of several allergy symptoms of shortness of breath, dizziness and itching, which were controlled partially with oral antihistamines, prednisolone and maintaining the same dose until clear. Finally, after 12 weeks, the patient was able to tolerate pharmaceutical doses of Montelukast at 10 mg safely and managed to gain a better asthma control.

Conclusion: To our knowledge, this is the first case report of effective oral Montelukast desensitization in an asthmatic patient with hypersensitivity type 1 to Montelukast. Although such allergic reaction to antileukotrines is rare, it should be recognized by healthcare providers. If no alternative therapy is available to control asthma symptoms, oral desensitization could stand as a valid effective therapeutic option.
\end{abstract}

\section{Introduction}

Montelukast Sodium is a selective leukotriene receptor antagonist (LTRAs) that inhibits the leukotriene receptor. It is widely prescribed as an orally active asthma treating agent. Usually, it is well tolerated by almost all asthmatics with rare adverse reactions [1].

One of these adverse reactions is hypersensitivity which is reported in few cases [1]. The mechanism of hypersensitivity to Montelukast is mainly a type I hypersensitivity (IgE antibody mediated) [2]. Literature review revealed at least three main patterns of type I hypersensitivity to Montelukast: 1) generalized urticarial rash, 2) angioedema [3] and 3) anaphylaxis [4]. Churg-strauss Vasculitis also has been documented as a complication to Montelukast [5].

The confirmation of clinically suspected type I hypersensitivity to a drug is mainly based on detailed clinical history by the constellation of exposure, timing, and clinical features [6]. In vivo (skin tests) and/or in vitro IgE tests (specific serum IgE antibodies) to drugs can also be used [7]. Unfortunately, there is no standard skin test to Montelukast as the literatures elucidate.

In most cases with drug allergy, the management is usually based on medications such as antihistamines and corticosteroids; avoidance of the component which caused the reaction, and switching to other alternatives [8]. However, when there are no other alternatives available in the market and therapy is mandatory with this drug, the use of an oral desensitization procedure with gradually increasing doses would be a good plan [8]. Subsequently, the re-use of therapeutic doses can be achieved safely [7]. Desensitization procedure needs a close up monitoring of the patient for any side effect or allergic reaction which may need immediate action [7].
The aim of this case report is to sort out this patient problem with Montelukast type I hypersensitivity, to evaluate the response to the desensitization procedure to Montelukast and to document this for the first time in the literature.

\section{Material and Method}

\section{Case history}

This is a 30 -years-old Saudi woman diagnosed with asthma, controlled on Budesonide/Formoterol turbohaler, one puff BID plus Montelukast $10 \mathrm{mg}$ once daily. Her asthma started since childhood and her chest symptoms started to become more recurrent when she was 6 years old. Her chest symptoms include a shortness of breath, cough, wheeze and yellowish sputum. These symptoms become worse in early morning, after flu and during winter. Her chest symptoms continue throughout the year.

Systemic review showed no (haemoptysis, fever, rigors, heartburn,

*Corresponding author: Moufag Mohammed Saeed Tayeb, King Abdul-Aziz University, Jeddah, Saudi Arabia, E-mail: moufagta@yahoo.com

Received November 20, 2012; Accepted December 30, 2012; Published January 04, 2013

Citation: Tayeb MMS (2013) Allergy to Montelukast Sodium Treated Effectively by Protracted Oral Desensitization: First Case Report. J Aller Ther 4:129. doi:10.4172/2155-6121.1000129

Copyright: (C) 2013 Tayeb MMS . This is an open-access article distributed unde the terms of the Creative Commons Attribution License, which permits unrestricted use, distribution, and reproduction in any medium, provided the original author and source are credited. 
orthopnea, hyperventilation nor paroxysmal nocturnal dyspnea). No history of smoking nor hypertension. On examination, vital signs were normal. No use of accessory respiratory muscles. Also, there was no (eye pallor, cyanosis, clubbing, decreased air entry, basal crepitations nor lower limb swelling). Chest X-ray was negative.

She is also a known case of chronic rhinitis which had started since puberty, controlled on Mometasone Furoate nasal spray, once daily. Her rhinitis was complicated into rhinitis medicamentosa due to the frequent use of Xylometazoline nasal drops. She is also a case of mild atopic dermatitis, subclinical hypothyroidism and vitamin D deficiency.

Unfortunately, five month after using Montelukast, she developed a recurrent itching and skin rash which disappeared after stopping Montelukast. Re-exposure to Montelukast within two months caused an immediate lip swelling, skin rash and shortness of breath. Montelukast was stopped and Chlorpheniramine Maleate, $4 \mathrm{mg}$ tablet was prescribed to control her allergic symptoms.

Subsequently, her asthma symptoms control deteriorated after stopping the Montelukast. An alternative manufacturer for Montelukast such as $\left(\right.$ Romilast $\left.^{\circledR}\right)$ Zafirlukast is a good option to try but unfortunately it's not available in the market of Saudi. Then, she was referred from her family physician to the allergy clinic for a proper asthma control.

At the private allergy clinic at King Abdulaziz University Hospital (KAUH) in Jeddah, her clinical data was revaluated and detailed history was taken. Asthma in this patient was classified by severity as severe persistent according to the British Thoracic Society guideline (BTS) [9]. Also it was classified by control as uncontrolled according to the Global Initiative for Asthma guideline (GINA) [1]. Skin prick test to common food and inhalant allergens was negative.

Anti-asthma medications were used in a stepwise approach at (step 4) as the maximal dose according to (GINA) guideline [1]. Budesonide/ Formoterol turbohaler were prescribed as two puffs BID plus if needed. Theophylline half tablet nocte, Prednisolone $5 \mathrm{mg}$ tablets course, and Mometasone Furoate nasal spray once daily. Other drugs added were: thyroxin tablet $25 \mathrm{mcg}$ OD and one alpha tablet $0.25 \mathrm{mg}$ OD.

Next visit within two weeks showed that her total serum IgE was high at $505 \mathrm{IU} / \mathrm{ml}$, negative IgE antibody screen to common inhalant allergens (Phadiatop ${ }^{\circledR}$ ), normal total Eosinophil count at $0.12 \mathrm{~K} / \mathrm{ul}$, negative RAST to common twenty food and twenty inhalant allergens.

However inspite of high IgE level, the diagnosis of allergic asthma isn't clear in this patient and it was classified as non-allergic type. That's why anti IgE Omalizumab was not an option plus its commercial impact and the frequent subcutaneous injections which were not accepted by the patient.

She was diagnosed with uncontrolled, non allergic asthma and rhinitis with type I hypersensitivity to Montelukast. Type I IgE hypersensitivity to Montelukast was verified by the clear correlation of typical symptoms immediately after exposure. Since there is no single standardized diagnostic test to confirm Montelukast hypersensitivity, it is mainly diagnosed by the constellation of exposure, timing, and clinical features including the pattern of organ manifestations.

This patient was already on maximum anti-asthma medications except antileukotrines. Because no other alternatives were available to Montelukast and there was an obligatory need for the use of Montelukast for enhanced asthma control, desensitization to Montelukast was determined as an acceptable and safe tool of drug allergy treatment $[7,8]$.

\section{Intervention}

Since oral desensitization to Montelukast in this case will be carried out for the first time, the chosen desensitization protocol will depend on simulation to other protocols of oral desensitization to other drugs which was conducted successfully. Several protocols of oral desensitization to drugs were collected and evaluated. Examples of desensitization protocols, like the ultra-rush protocol, continuous subcutaneous insulin infusion (CSII) [10,11]. The protocol of oral desensitization through gradually increasing doses of Montelukast was opted for because it is safer to the patient.

The desensitization procedure and its potential risks were discussed with the patient and her family, and then an informed verbal consent was obtained from the patient. Oral suspension was prepared at the allergy private clinic at KAUH. Montelukast sachet $4 \mathrm{mg}$ was dissolved in distilled water at a concentration of $4 \mathrm{mg}$ per $\mathrm{ml}$. Oral desensitization to Montelukast was planned over two phases [12].

Phase I desensitization took place at the allergy clinic at KAUH over 3 hours. Desensitization was initiated at 7:00 pm on Tuesday, $7^{\text {th }}$ of February 2012 by using a very low oral dose of Montelukast as $0.01 \mathrm{mg}$, at a concentration of $0.004 \mathrm{mg}$ per $\mathrm{ml}$ diluted 3 times. The doses were increased gradually every 15 minutes and reached a dose of $1 \mathrm{mg}$ (Table 1). Throughout Phase I desensitization procedure, her vital signs were measured every 15 minutes and were within normal range.

The subsequent doses were taken at home in Phase II desensitization starting from $1 \mathrm{mg}$ to a target dosage of $10 \mathrm{mg}$ of oral Montelukast/day. She was instructed to continue on the same dose for 3 days if any mild reactions developed, and to take oral antihistamine Chlorpheniramine Maleate $4 \mathrm{mg}$ as needed. However, she was advised to prolong the period with the previous tolerated dose during the appearance of allergic symptoms as shown in table 2 . She was also prescribed a self-injectable epinephrine as an emergency treatment in case of any systemic reaction or anaphylaxis that might occur, and to seek emergency care immediately [13]. A close follow up by clinic visits, e-mails and phone calls was reserved for serious issues [14].

Allergic reactions that appeared first in phase I included shortness of breath and dizziness after taking the Montelukast suspension dose of $0.04 \mathrm{mg}$ which was recovered after single dose of oral antihistamine Chlorpheniramine Maleate $4 \mathrm{mg}$. Another allergic reaction of mild dizziness developed at dose $0.08 \mathrm{mg}$, and recovered after a single dose of oral antihistamine also.

Such allergic reactions re-occurred at phase II at home at doses of $1 \mathrm{mg}, 4 \mathrm{mg}, 7 \mathrm{mg}$ and the last one at $8.5 \mathrm{mg}$. Allergic reactions took a protracted course (up to 82 days) to disappear. Medications

\begin{tabular}{|l|l|l|l|l|l|}
\hline Min. & Dilution & $\begin{array}{l}\text { Conc. } \\
\mathbf{m g} / \mathbf{m l}\end{array}$ & Dose $\mathbf{m g}$ & $\begin{array}{l}\text { Volume } \\
(\mathbf{m l})\end{array}$ & Symptoms \\
\hline 0 & 3 & 0.004 & 0.01 & 2.5 & No symptoms \\
\hline 15 & 3 & 0.004 & 0.02 & 5 & No symptoms \\
\hline 30 & 2 & 0.4 & 0.04 & 0.1 & $\begin{array}{l}\text { Shortness of breath and dizziness. } \\
\text { Period prolonged to } 30 \text { minute to take } \\
\text { the next dose plus given antihistamine }\end{array}$ \\
\hline 60 & 2 & 0.4 & 0.08 & 0.2 & Mild dizziness \\
\hline 15 & 2 & 0.4 & 0.16 & 0.4 & No symptoms \\
\hline 30 & 2 & 0.4 & 0.32 & 0.8 & No symptoms \\
\hline 45 & 1 & 4 & 0.64 & 0.16 & No symptoms \\
\hline 60 & 1 & 4 & 0.8 & 0.2 & No symptoms \\
\hline 14 & 1 & 4 & 1 & 0.25 & No symptoms \\
\hline
\end{tabular}

Table 1: Desensitization Phase I. 


\begin{tabular}{|l|c|l|}
\hline Day & Dose $\mathbf{m g}$ & Symptoms \\
\hline $1-17$ & 1 & $\begin{array}{l}\text { Dyspnea, swelling of eye lids, itching, hoarseness and } \\
\text { tiredness relieved by Symbicort and antihistamine }\end{array}$ \\
\hline $18-20$ & 1.5 & No symptoms \\
\hline $21-23$ & 2 & No symptoms \\
\hline $24-26$ & 2.5 & No symptoms \\
\hline $27-29$ & 3 & No symptoms \\
\hline $30-32$ & 3.5 & No symptoms \\
\hline $33-46$ & 4 & $\begin{array}{l}\text { Congested throat, yellow sputum, shortness of breath, fever } \\
\text { relieved with Prednisolone, Mucolytic, Theophylline }\end{array}$ \\
\hline $47-49$ & 4.5 & No symptoms \\
\hline $50-52$ & 5 & No symptoms \\
\hline $53-55$ & 5.5 & No symptoms \\
\hline $56-58$ & 6 & No symptoms \\
\hline $59-61$ & 6.5 & No symptoms \\
\hline $62-64$ & 7 & Mild shortness of breath relieved by Symbicort \\
\hline $65-67$ & 7.5 & No symptoms \\
\hline $68-70$ & 8 & No symptoms \\
\hline $71-73$ & 8.5 & Mild itch relieved by antihistamine \\
\hline $74-76$ & 9 & No symptoms \\
\hline $77-79$ & 9.5 & No symptoms \\
\hline $80-82$ & 10 & No symptoms \\
\hline & & \\
\hline
\end{tabular}

Table 2: Desensitization Phase II.

advised were: Prednisolone $5 \mathrm{mg}$ tablets as needed, Theoped tablets nocte, Chlorpheniramine Maleate $4 \mathrm{mg}$ tablet as needed, Budesonide/ Formoterol turbohaler one puff as needed and Mometasone Furoate nasal spray once daily.

The total duration of Phase II desensitization process at home was 82 days as shown in table 2 . When the Montelukast doses reached 10 $\mathrm{mg} /$ day with no serious side effect, the dose was repeated for three days then the patient was advised to take the Montelukast tablets in the form of $10 \mathrm{mg}$. She was seen in a follow-up visit after the end of Phase II desensitization. Asthma symptoms were much better and the desensitization process was maintained at this dose.

Further follow up visits were arranged for any late reactions, and the final dose of Montelukast after successful desensitization was adjusted. The patient was told to comply strictly with the daily Montelukast, as any missed dose may lead to loss of tolerance and the need to repeat the desensitization process from the start.

There was a close monitoring throughout the desensitization procedure by patient visits to the clinic, phone calls and e-mails for reporting any side effects, answering any questions, giving advices and appropriate responses. Patient visit to the clinic was after one, three and six month. This contact was a very helpful way with many rewards to the patient and the team. Moreover, this helped create a closer relationship between the treating physician and the patient, improve the patient compliance, answer the patient concerns quickly, reassure the patient if any adverse reaction appears and involves the patient in supervising her own health care [14].

\section{Result}

Desensitization phases I was successful over a period of 3 hours with minimal reactions while phase II was prolonged due to the occurrence of several allergy symptoms. After 12 weeks, the patient was able to tolerate pharmaceutical doses of Montelukast at $10 \mathrm{mg}$ safely and asthma symptoms were controlled.

Our case had a successful first attempt of oral Montelukast desensitization. Apart from very mild symptoms that were resolved with oral medications, the desensitization protocol was completed successfully.

\section{Discussion}

Although the prevalence of type I hypersensitivity to oral Montelukast has been rarely reported, it can still happen as in this case report. Therefore, health care providers must keep in mind the possibility of Montelukast allergy when initiating such therapy. Physicians who prescribe Montelukast must be aware of anaphylaxis as the most serious complication even if its prevalence is rare [4].

There are few readily available alternatives for asthmatics that are intolerant to Montelukast in the international markets. They include agents such as Montelukast Sodium (Romilast ${ }^{\circledR}$ ), an alternative manufacturing company for Montelukast.

Desensitization to Montelukast poses potential risks, and is not recommended as a first-choice treatment for all patients with allergic reactions to the drug. The procedure is beneficial in selected patients who had allergic reactions to this medication, when other alternatives are not available, or in patients who cannot be treated with other antileukotrine drugs [8].

Montelukast desensitization protocols are not available as this is the first case report, that's why a slow protracted oral desensitization protocol has been selected as a safe and effective regimen. That is why it remains difficult to reach the exact success and failure rates of this protocol. Continued monitoring of patients, both during and following desensitization, is important as hypersensitivity to the drug may recur [7].

Electronic communications have been proven to be effective in facilitating communication among care providers and patients, thereby allowing for greater continuity of care and more timely interventions. E-mail communications for close follow up can be a very helpful tool for a 24 hours monitoring of patients progress without the need to come to the clinic [12].

\section{Conclusion}

Type I hypersensitivity to Montelukast can occur but rarely; results from the present study confirm the efficacy and the safety of slow protracted oral desensitization to Montelukast.

It is crucial to do Montelukast desensitization after trying all the other tools because of anaphylaxis risk with desensitization procedure. It's vital to exclude all the diseases which could be similar to asthma. The desensitization procedure can be carried out in an outpatient setting under close medical supervision after careful counseling and detailed discussions with the patient prior to initiating therapy [15]. The new intervention in this case report is the use of a slow protracted protocol desensitization process. To our knowledge, this is the first case report in literature. Montelukast is well tolerated with a safety profile that is similar in adult and pediatric populations [16].

\section{Acknowledgment}

I would like to extend my heartfelt gratitude to Prof. Emad Koshak (Consultan Internal Medicine, Allergy \& Clinical Immunology, Dean Faculty of Medicine-Albaha University-Kingdome of Saudi Arabia), Dr. Khalid Bawakid (Consultant, Ministry of Health-Jeddah-Saudi Arabia) and Dr. Majdy Qutub (Consultant, King Abdu Aziz University Hospital-Jeddah-Saudi Arabia) for their support and assistance in revising this case report, analyzing the data and providing highly efficient suggestions. Prof. Koshak was my supervisor throughout all the clinical process with this patient. I feel proud to be a member of this team that has always been there for me and helped me all the way through the writing of this case report. 
Citation: Tayeb MMS (2013) Allergy to Montelukast Sodium Treated Effectively by Protracted Oral Desensitization: First Case Report. J Aller Ther 4:129. doi:10.4172/2155-6121.1000129

\section{References}

1. Bateman E, Boulet LP, Ohta K, Cruz A, Paggario P, et al. (2011) Global initiative for Asthma. GINA guideline.

2. Minciullo PL, Saija A, Bonanno D, Ferlazzo E, Gangemi S (2004) Montelukastinduced generalized urticaria. Ann Pharmacother 38: 999-1001.

3. Sabbagh R, Sheikh-Taha M (2009) Possible montelukast-induced angioedema. Am J Health Syst Pharm 66: 1705-1706.

4. Gerard A, Harkisoon S (2009) Singulair-induced anaphylaxis? J Fam Pract 58: 133-134

5. Kaliterna DM, Perkovic D, Radic M (2009) Churg-Strauss syndrome associated with montelukast therapy. J Asthma 46: 604-605.

6. Riedl MA, Casillas AM (2003) Adverse drug reactions: types and treatment options. Am Fam Physician 68: 1781-1790.

7. Mirakian R, Ewan PW, Durham SR, Youlten LJ, Dugué P, et al. (2009) BSAC guidelines for the management of drug allergy. Clin Exp Allergy 39: 43-61.

8. Cernadas JR, Brockow K, Romano A, Aberer W, Torres MJ, et al. (2010) General considerations on rapid desensitization for drug hypersensitivity-a consensus statement. Allergy 65: 1357-1366.

9. (2012) British Guideline on the Management of Asthma. A national clinical guideline 101 .
10. Hasselmann C, Pecquet C, Bismuth E, Raverdy C, Sola-Gazagnes A, et al. (2012) Continuous subcutaneous insulin infusion allows tolerance induction and diabetes treatment in a type 1 diabetic child with insulin allergy. Diabetes Metab.

11. Pföhler C, Müller CS, Hasselmann DO, Tilgen W (2008) Successfu desensitization with human insulin in a patient with an insulin allergy and hypersensitivity to protamine: a case report. J Med Case Rep 2: 283.

12. Tayeb M, Koshak E, Alhadramy M (2008) Successful Dual Desensitization with Biphasic Isophane Insulin (Mixtard $® 30$ ) for Insulin Allergy in a Diabetic Patient. Tanta Medical Sciences Journal.

13. Topal E, Bakirtas A, Yilmaz O, Ertoy IH, Arga M, et al. (2012) A Real-Life Study on Acquired Skills from Using an Adrenaline Autoinjector. Int Arch Allergy Immunol 160: 301-306.

14. Bawakid KO, Qutub M, Tayeb M, Koshak K (2011) Application of e-mail in Successful Desensitization to Allopurinol. A Case Report. Saudi Society of Internal Medicine 1.

15. Hugh S (2004) Out of Hours telephone consulting by nurses and doctors. BMJ

16. Benninger MS, Waters $\mathrm{H}$ (2009) Benninger and Heather waters. Montelukast: Pharmacology, Safety, Tolerability and Efficacy. Clinical Medicine Insights: Therapeutics 1: 1253-1261. 